\title{
Zur Vita der hl. Theodora von Thessalonike.1)
}

Den Sammelcodex der Moskauer Synodalbibliothek früher Nr. 159, jetzt (nach einer Notiz von Arsenij) 390 hatte schon im Jahre 1896 Vasiljevskij ausführlich besprochen und daraus auch reichliche Auszüge der vita Theodorae gegeben; s. Kurtz, Byz. Z. II (1893) S. 312-313. Jetzt liegt vor uns das ganze wertvolle Leben nach demselben Codex herausgegeben; leider ist aber der Text desselben ohne den nötigen kritischen Kommentar gedruckt ${ }^{2}$ ), sodals man in korrupten Stellen nicht wissen kann, was der Hs selbst und was dem Herausgeber zu verdanken ist. Ich begnüge mich also mit folgenden wenigen Emendationen.

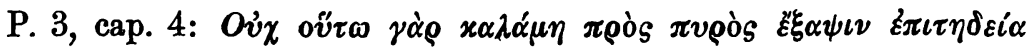

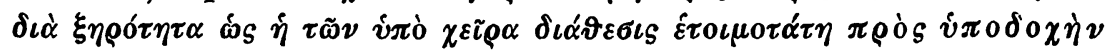

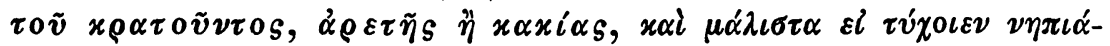

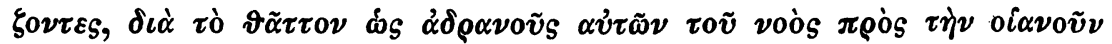

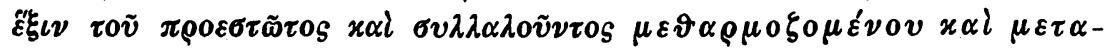
$\pi i \pi \tau 0 \nu \tau o s$.

Vielleicht hat sich der Verfasser selbst so ausgedrückt, vielleicht aber auch der Schreiber geirrt, indem er die Inf. $\mu \varepsilon \vartheta \alpha \rho \mu \delta \xi \varepsilon \sigma \vartheta \alpha \iota$ und

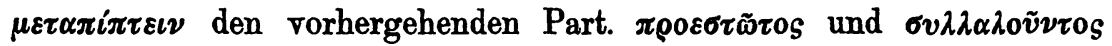

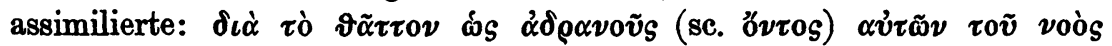

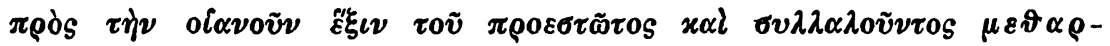

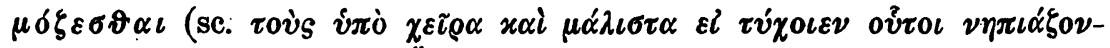
$\tau \varepsilon s) x \alpha i \mu \varepsilon \tau \alpha \pi i \pi \tau \varepsilon \iota \nu$. Übrigens ist das Komma nach $x \rho \alpha \tau o v v \tau o s \mathrm{zu}$

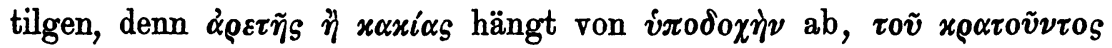

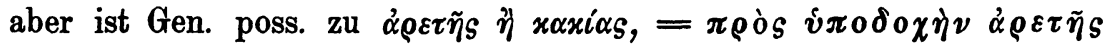
$\ddot{\eta} x \alpha x i \alpha S \tau o \tilde{v} x \rho \alpha \tau o \tilde{v} \nu \tau o s$.

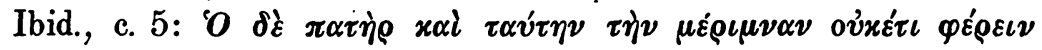

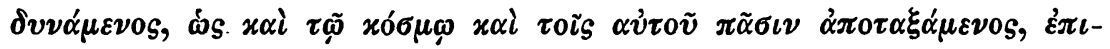

1) Bisch of Arsenij, Leben der hl. Theodora von Thessalonike. Griechischer Text (pag. 1-36) mit russischer Übersetzung (p. 37-79). 1899.

2) Die Bibelstellen sind mitten im Texte der Übersetzung angezeigt. 


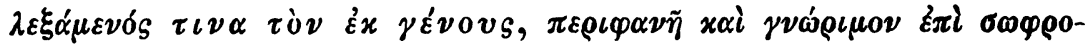

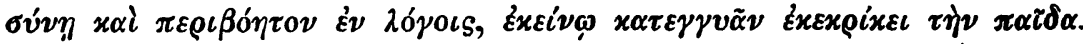

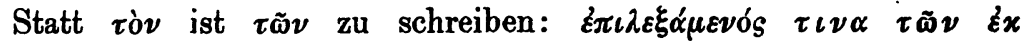

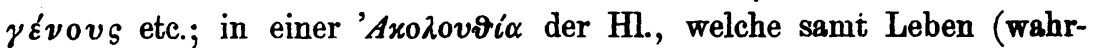

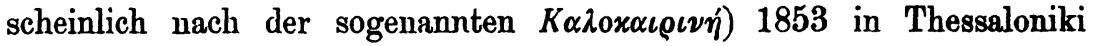

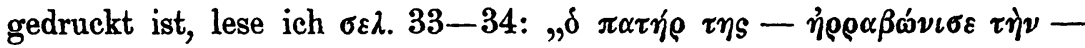

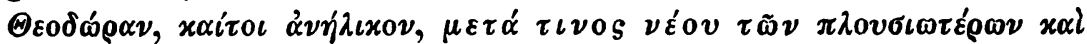

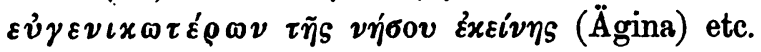

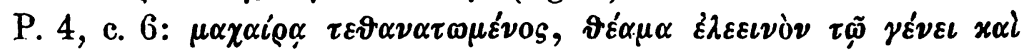

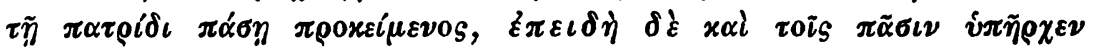

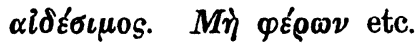

Schr. $\dot{\varepsilon} \pi \varepsilon \iota \delta \dot{\eta}[\delta \dot{\delta} \xi x \alpha i$ etc.

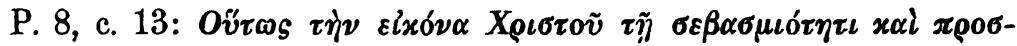

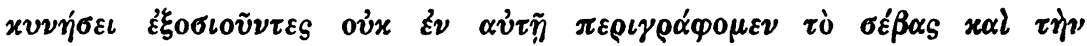

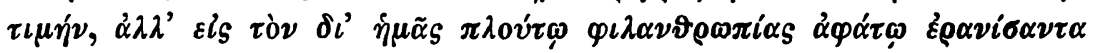

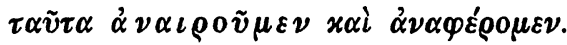

St. des widersinnigen $\alpha \dot{\alpha} \alpha \iota \rho o \tilde{v} \mu \varepsilon v$ ist wohl $\alpha \nu \alpha i \varrho o \mu \varepsilon v$ zu setzen.

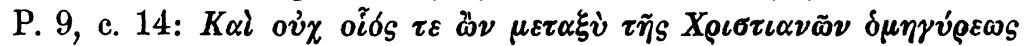

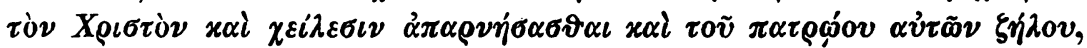

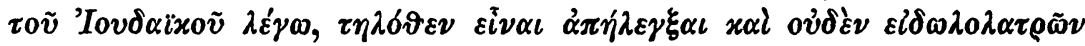

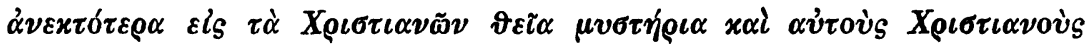

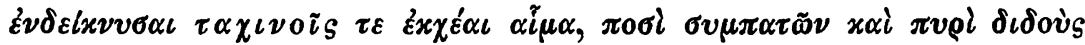

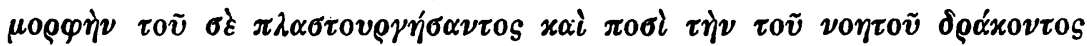

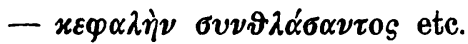

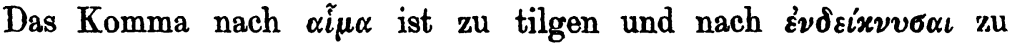

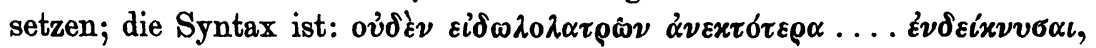

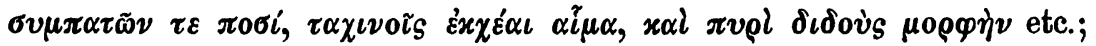

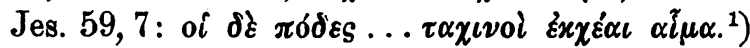

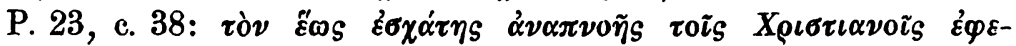

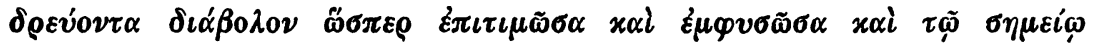
$\sigma \tau \alpha v \varrho \circ \tilde{v} x \alpha \tau \alpha \rho \gamma \tilde{\omega} \sigma \alpha \varepsilon^{\prime \prime} \lambda \varepsilon \gamma \varepsilon \nu$ etc.

Das unerhörte $x \alpha \tau \alpha \rho \gamma \tilde{\omega} \sigma \alpha$ (st. $x \alpha \tau \alpha \rho \gamma \sigma \tilde{v} \sigma \alpha$ ) ist vielleicht unter dem Einflusse von $\dot{\varepsilon} \pi \iota \tau \iota \mu \tilde{\omega} \sigma \alpha$ und $\dot{\varepsilon} \mu \varphi v \sigma \tilde{\omega} \sigma \alpha$ geschrieben.

Neue Wörter habe ich mir angemerkt:

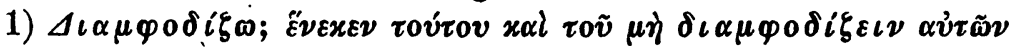

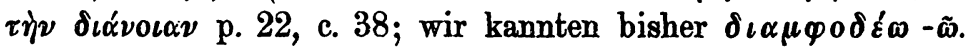

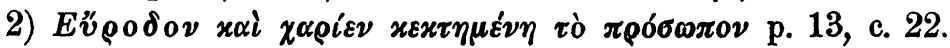

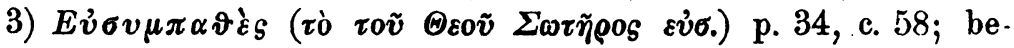
kannt ist $\varepsilon \dot{v} \sigma v \mu \pi \alpha^{\prime} \vartheta \eta \tau \sigma$ s.

1) Die Notiz der Bibelstelle verdanke ich meinem Freunde Hr. Kurtz. 


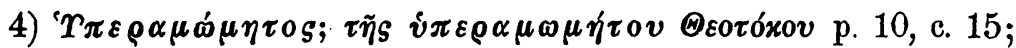

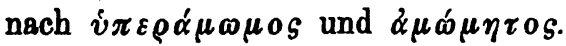

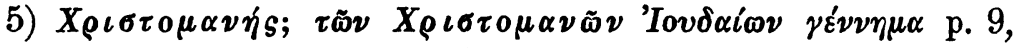
c. 14; Sophocles, Greek Lexicon (N. York 1887) p. 1172 und Kumanudis $\sigma \varepsilon \lambda .379$ führen $X_{\varrho} \iota \sigma \tau \mu \alpha \nu i \alpha$ an.

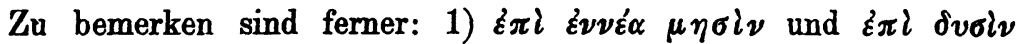

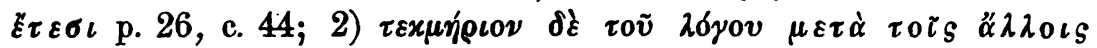

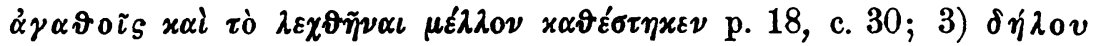

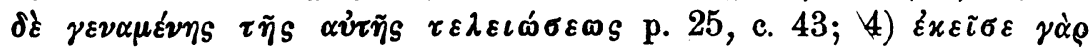

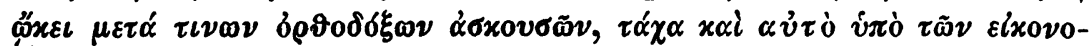

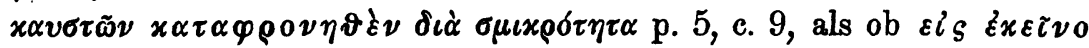

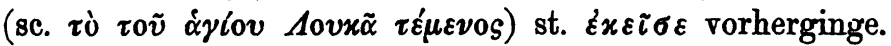

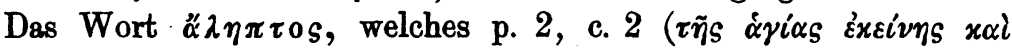
$\ddot{b} \nu \tau \omega s \dot{\alpha} \lambda \dot{\eta} \pi \tau$ ov $\psi v \chi \tilde{\eta} s)$ vorkommt, hat mich an die Stelle des Eucho-

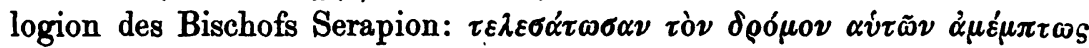

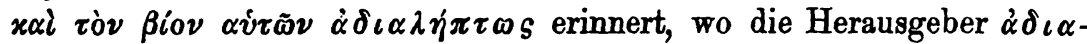
$\lambda \varepsilon i \pi \tau \omega s$ schreiben, mein Freund Hr. Kurtz, Byz. Z. VIII (1899) 646, $\dot{\alpha} \delta\llcorner\alpha \beta \lambda \eta \tilde{\tau} \omega s$ vorschlägt; vielleicht ist $\dot{\alpha} \delta \iota \alpha \lambda \dot{\eta} \pi \tau \omega s$ ein verstärktes $\dot{\alpha} \lambda \dot{\eta} \pi \tau \omega s$, welches Hesychios (M. Schmidt ed. mai. I p. 119, 2944) durch à $x \alpha \tau \alpha \gamma \nu \omega ́ \sigma \tau \omega s$ erklärt; ist meine Ansicht richtig, so kann man gestrost das Adv. $\alpha \delta \iota \alpha \lambda \eta \dot{\eta} \pi \omega_{S}$ in die Lexika eintragen.

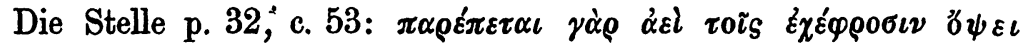

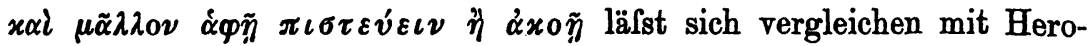

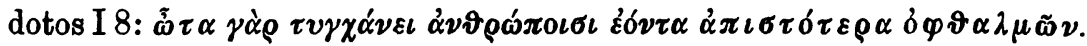

Die vita ist von grofser Bedeutung für die Topographie von Thessalonike im 9. Jahrh., wie es in der Byz. Z. a. a. O. angedeutet ist; indem ich mir reichlichere Mitteilungen aus meinen topographischen Studien über die Stadt vorbehalte, bemerke ich hier kurz nur Folgendes.

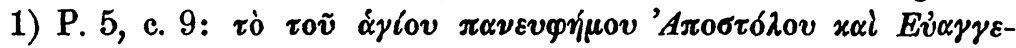

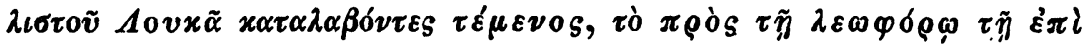

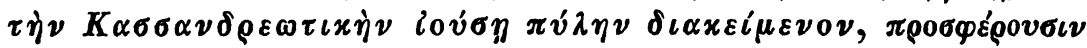

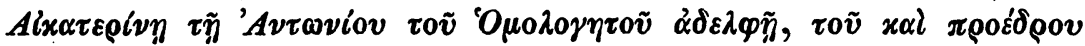

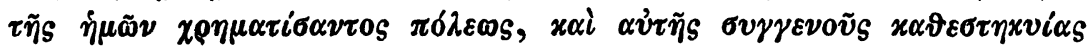

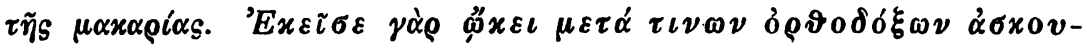

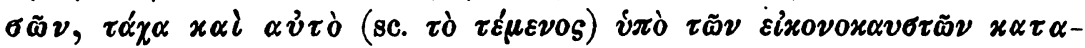
$\varphi \rho 0 \nu \eta \vartheta \grave{\varepsilon} \nu \delta \iota \dot{\alpha} \sigma \mu \iota x \rho \sigma ́ \tau \eta \tau \alpha$.

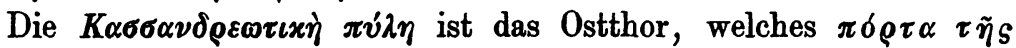
$K \alpha \lambda \alpha \mu \alpha \rho\lfloor\tilde{\alpha} s$ heifst (Tafel de Thessalonica p. 99 und $M \iota \chi \alpha \dot{\eta} \lambda X \alpha \tau \zeta \tilde{\eta}$

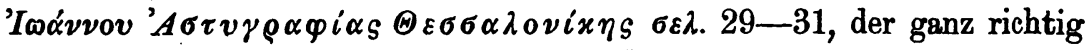

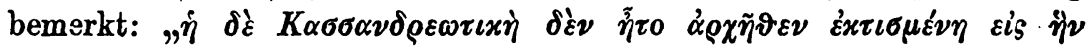

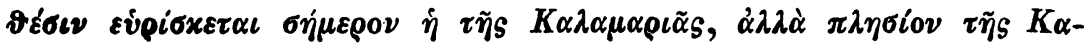




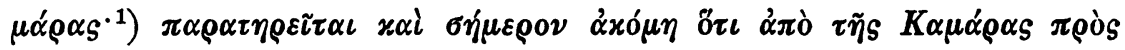

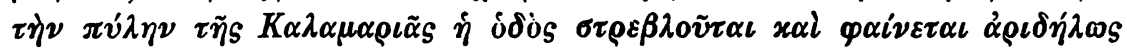

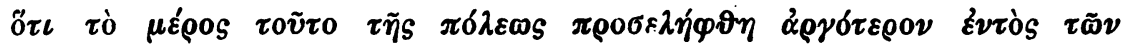
$\tau \varepsilon \iota \chi \tilde{\omega} \nu$. "2) Durch $\lambda \varepsilon \omega \varphi o ́ \rho \circ \varsigma$ bezeichnet der Biograph zweifellos die via Egnatia (jetzt $\mu \varepsilon \gamma \alpha \dot{\lambda} o_{s}$ oder $\left.\varphi \alpha \rho \delta \dot{v} s \delta \rho \delta \mu o s\right)$. Das Kloster, in welchem Äkaterine (nach der erwähnten Akoluthia $\sigma \varepsilon \lambda .34$ eine

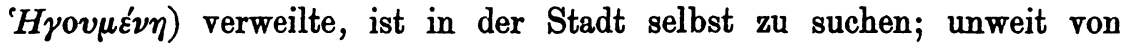

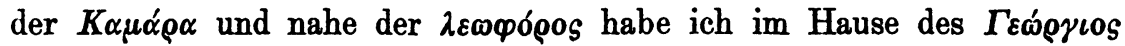

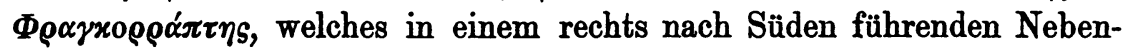
wege liegt, ein niedergerissenes $\pi \alpha \rho \varepsilon x x \lambda \eta \dot{\sigma} \sigma \nu \nu$ mit einem alten Bilde des Evangelisten Lukas gefunden. Die Tradition, dafs dort ein Lukaskloster lag, wird durch die benachbarte Kirche der $\Pi \alpha v \alpha$ jov́d $\alpha$ bestätigt: von den zwei Nebenchören dieser Kirche nämlich trägt der eine (rechts) den Namen Seraphim, der andere (links) denjenigen von

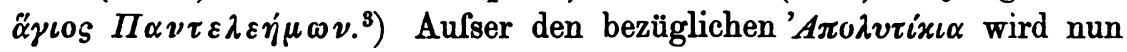
in der Kirche noch ein drittes, dasjenige über Lukas vorgetragen, sicher aus frommer Erinnerung an das einst naheliegende Kloster, wenn dies nicht vielmehr am Orte der jetzigen Panagudakirche gelegen hat. Im Exonarthex der genannten Kirche sieht man sechs alte Säulen und Kapitäle stehen, im Inneren zwei marmorene (Grab-)Platten am Boden liegen; sie tragen geflügelte Thiere, welche unter ihren Füfsen andere, habichtköpfige Tiere festhalten. Folgendes Stück ist im Triantaphyllos-Hause (aus dem 16. Jahrhundert), welches gegenüber dem östlichen Hofeingange der Kirche (in dem Nebenwege) liegt, dicht unter dem hohen Dache eingemauert; es entging meinen Augen bis auf den 16. Oktober d. J.:

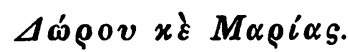

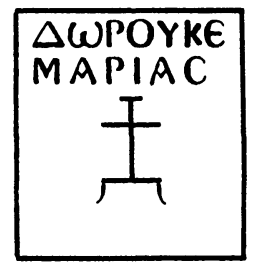

2) Nach dem Tode ihres Gatten tritt Theodora in ein Kloster ein:

1) K. Kinch, L'arc de triomphe de Salonique, Paris 1890.

2) Auch andere, sehr wichtige Gründe bestätigen diese Ansicht des Gelehrten, welcher der Wissenschaft zu früh entrissen worden ist.

3) Den Grund dazu lehrt die Nachbarschaft der nördlich, durch die $\lambda \varepsilon \omega \varphi$ óos getrennt liegenden schönen byzantinischen Kirche des Hagios Panteleïmon, jetzt Isakié-Djamissí (Texier, Descr. de l' As. min. III p. 62 „SankiéDjami-si"). 


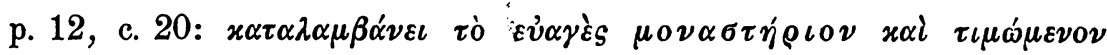

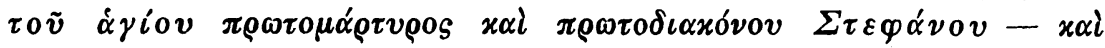

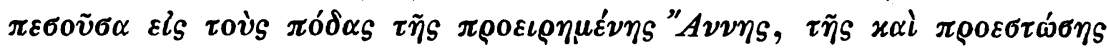
$\tau \tilde{\eta}_{S} \alpha \dot{v} \tau \tilde{\eta} S \mu o \nu \tilde{\eta} s, \varepsilon \lambda \varepsilon \gamma \varepsilon \nu$ etc.

Auch dies weibliche Kloster lag in der Stadt, und zwar sicherlich in demselben Orte, in welchem jetzt die Kirche der $\dot{\alpha} x i \alpha \Theta \varepsilon \delta \delta \omega ́ \rho \alpha$ liegt (unweit und westlich von der Kirche der Hagia Sophia, in einem fast ganz jüdisch gewordenen Quartier), welche in türkischen Urkunden Kizlár-Manastir, d. i. Weiberkloster, heifst; der Nebenchor rechts ist nach dem Protomartys Stephanos, derjenige links nach dem hl.

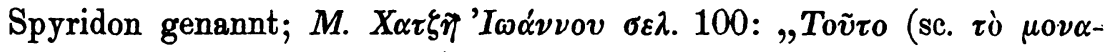

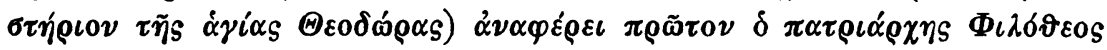

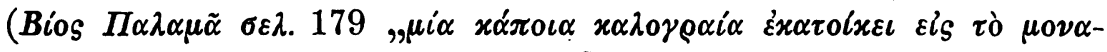

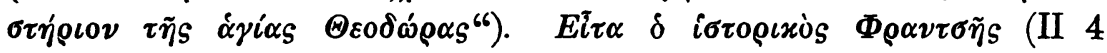

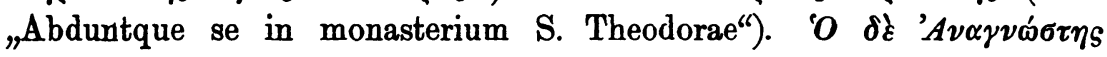

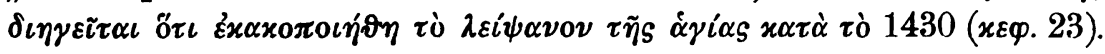

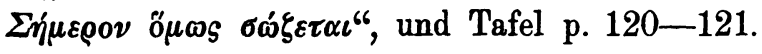

Aufser vielen alten architektonischen Marmorstücken (sechs Säulen, zwei Kapitälen, gezierten Grabplatten etc.), welche im Exonarthex und im Hofe der tief liegenden Kirche liegen, Bildern und anderen Sachen vom vorigen Jahrhundert, sind erhalten: 1) eine Pergamenthandschrift,

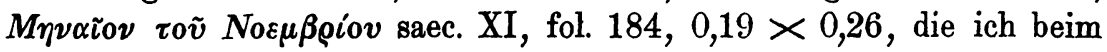
Priester Georgios gesehen habe (aufgefunden im Jahre 1880 im Dache der Kirche); 2) ein silbernes Kistchen mit verschiedenen Reliquien samt Namen der bezüglichen Persönlichkeiten (es gehörte einst einem deI von Abdoùl-Aboùt Pascha geplünderten Klöster, Byz. Z. VII, 1898 S. 61, und wurde nach einer Notiz in einem Codex auf Kosten der Kirche der hl. Theodora in Thessalonike gekauft); 3) Inschriften.

a) Kolossale Marmorplatte mit schön herausgearbeiteten, Rand-

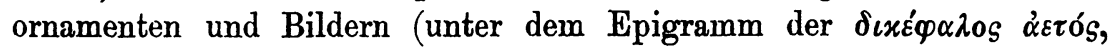
im Kreis, und Adam und Eva samt Baume, zwei Schlangèn und dem schlangenköpfigen Teufel, im Paradiese). Die Buchstaben der 15 Zeilen zählenden Inschrift sind leider von den Füfsen ganz abgerieben; was ich herauszulesen vermochte (1889 Sept. und 1898 Aug.), ist Folgendes:

\section{Z. $1 \times$ ir}

Z. 11 ГCPON' CCTO¥IEPG'T KPY $\Psi$

\section{CY}

$\oiint^{\sharp} 3^{\omega}$
$110 \mathrm{C}$

MINI
ENANTTIOS

CAIIE $\Psi$ loC NO 


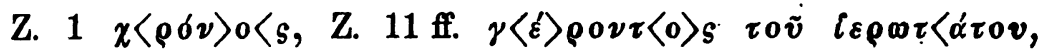
$x \rho v \psi\left\langle\varepsilon \nu, \varepsilon \dot{v} \alpha \nu \tau i o s, \mu\langle\eta\rangle v i, \dot{\alpha}\langle\nu\rangle \varepsilon \psi \iota o^{g}\right.$.

b) Würfelartiger Marmor (0,21 h., 0,17 br.) mit herausgeschnitztem, unten in zwei Blätter ausgespalteten Kreuze auf der Vorderseite; darüber

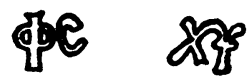

$\Phi(\tilde{\omega})$ s $X(\rho \iota \sigma \tau 0) \tilde{v}$. Das .Stück scheint einst Kapitäl gewesen zu sein; das erste Wort

$$
\Phi \omega
$$

habe ich auf einer Säule im Hofe der Pantokratorkirche ${ }^{1}$ ) und auf einer anderen in der Gottesmutterkirche (Eski Djouma-Djamissí) gesehen.

c) Die Reliquien der hl. Theodora (geb. 812 auf der Insel Ägina,

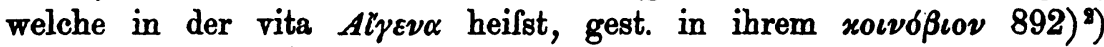
werden jetzt in einem marmorenen Sarge (0,35 h., 128 l., 0,65 br.) aufbewahrt (in dem Nebenzimmer des hl. Stephanos), dessen lange Vorderseite reich ausgeschmückt ist; die eine von den kleineren Nebenseiten (links) trägt folgende Inschrift in Relief mit verschiedenen Ligaturen und Kompendien, welche die Lesung sehr schwierig machen (1892 Juli):

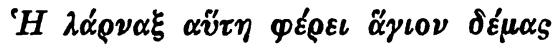

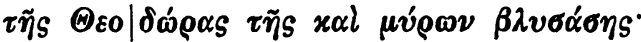

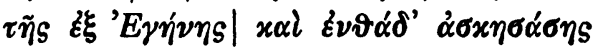

$\mu \varepsilon \tau \omega \varkappa \eta \sigma \tau \tilde{\eta} \sigma \alpha$ (sic) $\mu \varepsilon \tau \dot{\alpha} \mid \tau \tilde{\omega} \nu$

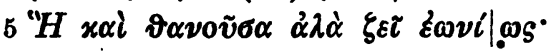

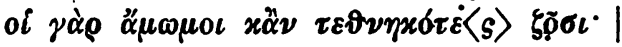

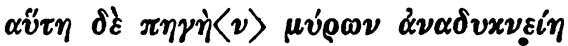

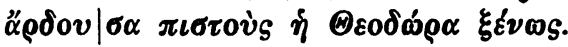

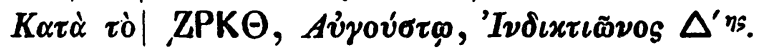

Z. 5 (V: 6) und Z. 6 (V. 7) sind die Buchstaben C und H (s und v) vom Steinmetz vergessen. Die Worte $\mu \varepsilon \tau \dot{\alpha} \tau \tilde{\omega} \nu$ gemeiner gemeint, denn nur der Vater kam nach Thessalonike mit, die Mutter war schon todt (p. 2, c. 3 und p. 4, c. 7).

Im Hofe der Kirche rechts zeigte man ein $x \alpha \rho \varepsilon x<\lambda$ y

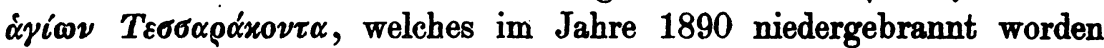

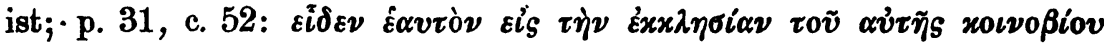

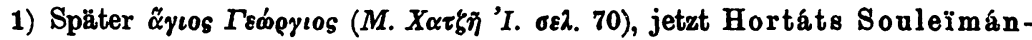
effendi Djamissí, bekannt in den Reisebüchern unter dem Namen Rotonde; der veraltete Unsinn von einem Kabirentempel (!) wird noch im Jahre 1896 bei

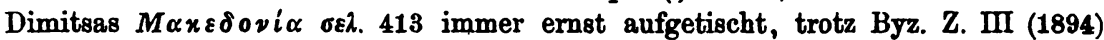
S. 248 Anm.

2) Ihr Andenken wird in Thessalonike nicht am 29., sondern am 3. August

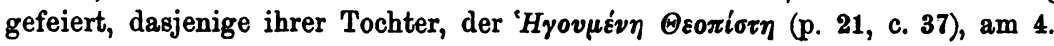




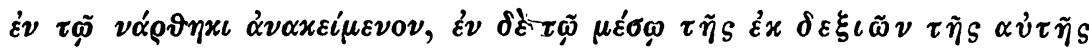

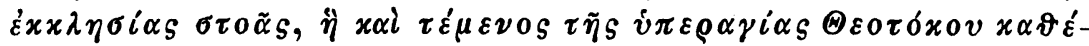

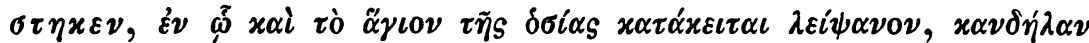

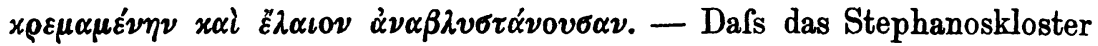
in der Stadt lag, beweist auch die vita, p. 14, c. 23: $x \alpha i \tau \eta \dot{\nu} \alpha \lambda \lambda \lambda \eta \nu$

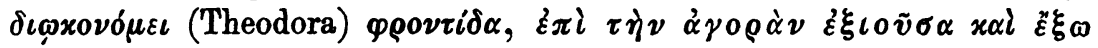

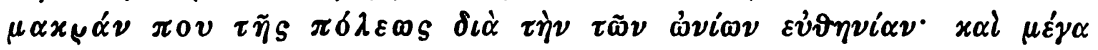

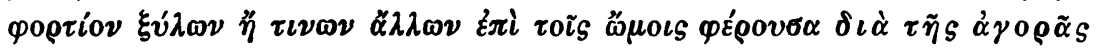
$\mu \varepsilon \varepsilon^{\prime} \sigma о \nu \varepsilon^{\prime} \beta \alpha^{\prime} \delta \iota \xi \varepsilon \nu$; gerade in der Nähe der Kirche fängt noch heute der Markt an. Damit steht in keinem Widerspruche p. 1, c. 1: $\dot{\varepsilon} \pi \varepsilon i$ oṽv

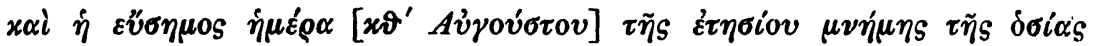

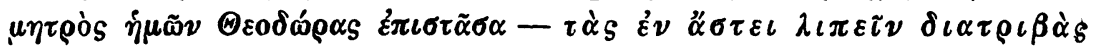

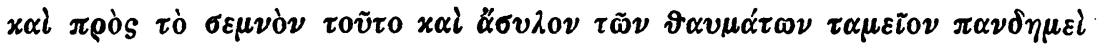

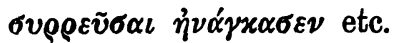

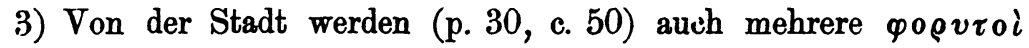

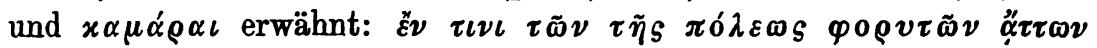

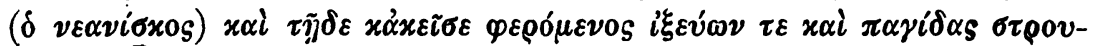

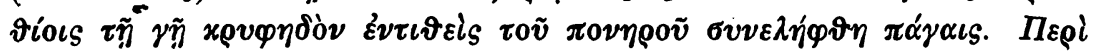

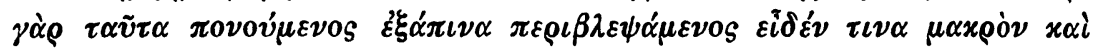

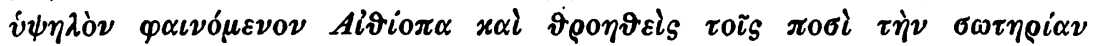

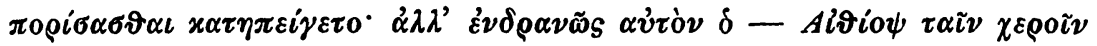

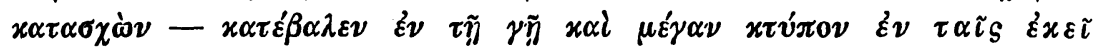

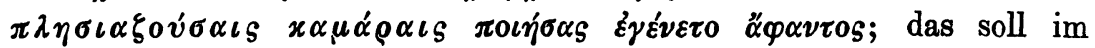

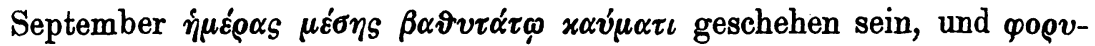

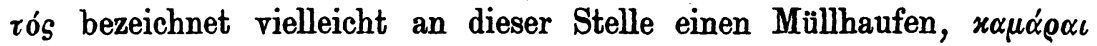
sind aber höchstwahrscheinlich Wasserleitungsbogen (einige von denselben, aufserhalb der Westmauer, tragen noch heute den türkischen

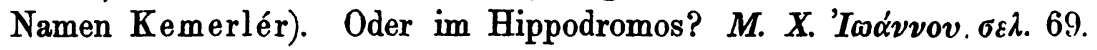

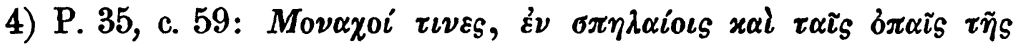

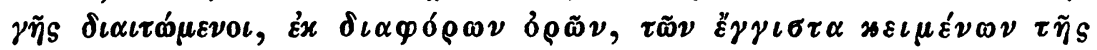

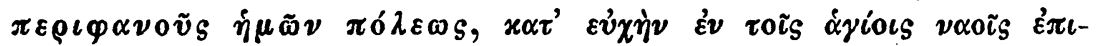

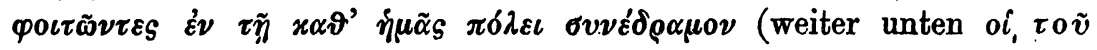

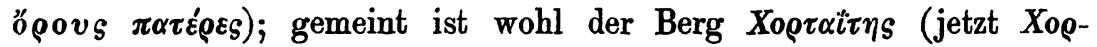
$\tau\lfloor\alpha ́ \tau \eta$ s) mit seinem einst berühmten, heute in sparsamen Trümmern

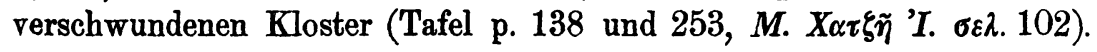

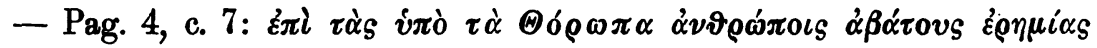
$\dot{\alpha} \pi \varepsilon \dot{\delta} \rho \alpha \mu \varepsilon$ (der Vater von Theodora) ist der Name vom Herausgeber in seinem kleinen Eigennamenregister übersehen worden.

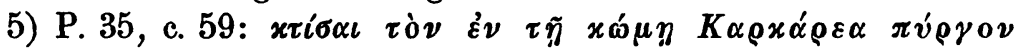

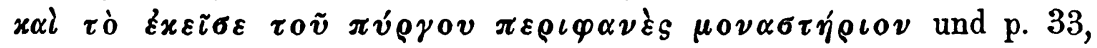

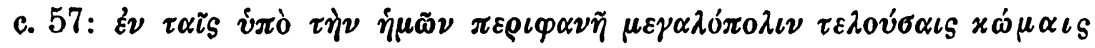




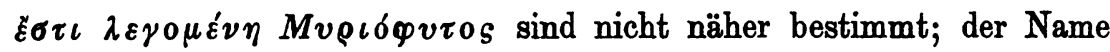

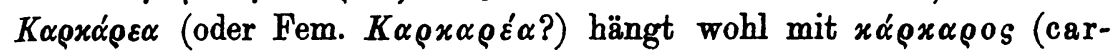

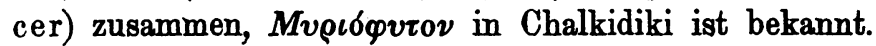

Nicht minder interessant sind die neuen historischen Notizen und die Persönlichkeiten, welche in der vita vorkommen.

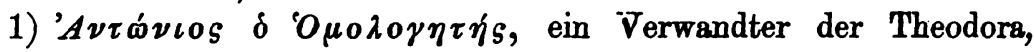

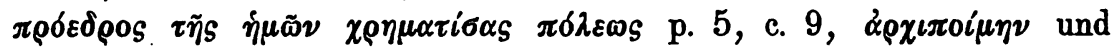

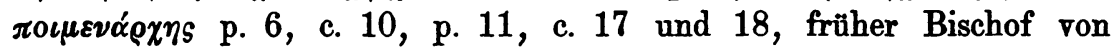
Dyrrhachion, mit welchem sich der Biograph c. 13-18 (p. 8-11) beschäftigt; er fehlt auch im Kataloge des Metropoliten von Amsssia, Anthimos Alexudis. ${ }^{1}$ )

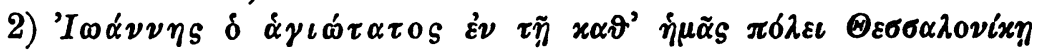

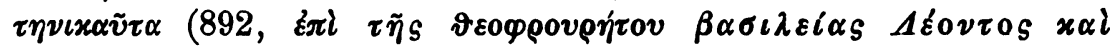

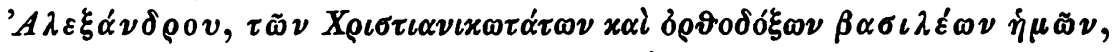

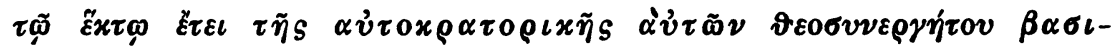

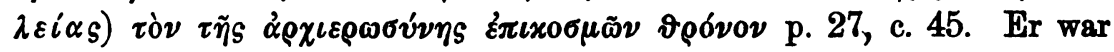
bisher nur durch folgende Inschrift bezeugt, die ich mit dem beigefügten Facsimile aus meinem unedierten Corpus mitteile. ${ }^{2}$ )

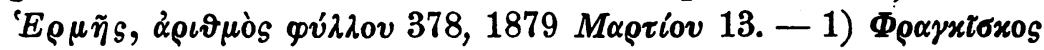

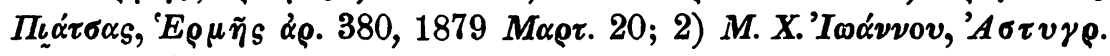
$\sigma \varepsilon \lambda .17$; 3) P. Papageorg(iu), Berliner philol. Wochenschr. 1883 Sp.

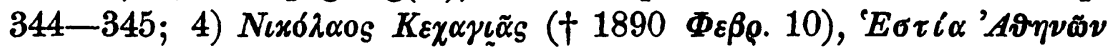

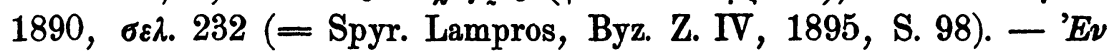

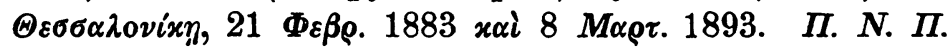

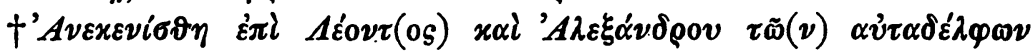

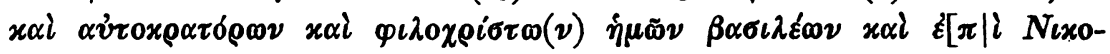

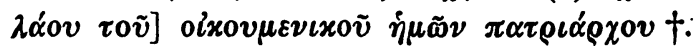

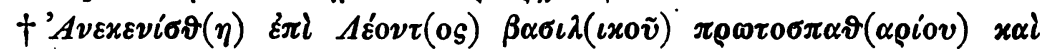

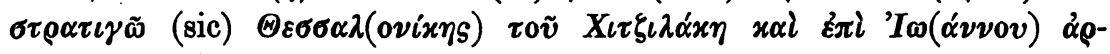

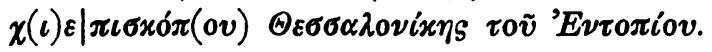

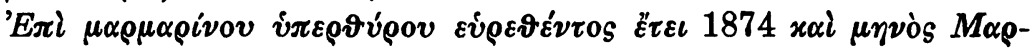

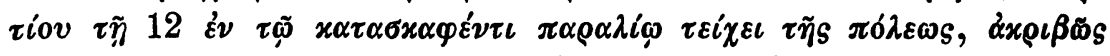

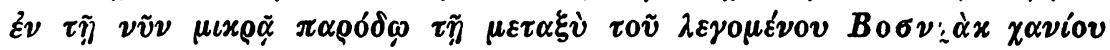

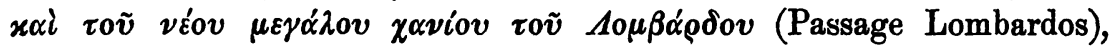

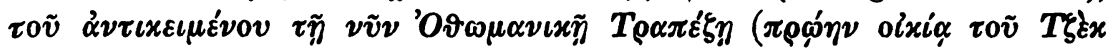

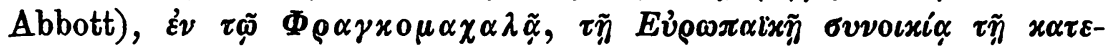

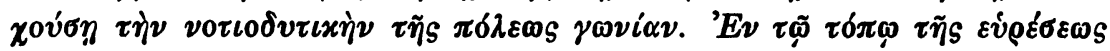

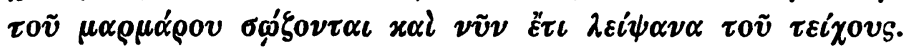

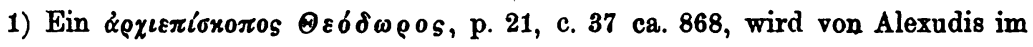
Jahre 879 angesetzt.

2) Ein 'I $\omega \alpha \nu \nu \eta s$ (derselbe?) wird bei Alexudis 880 angegeben. 


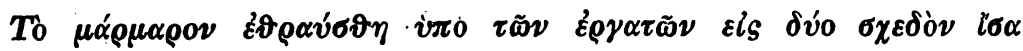

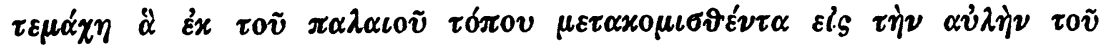

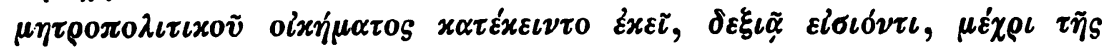

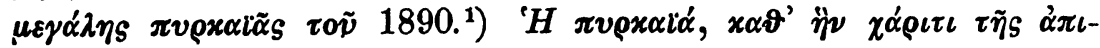

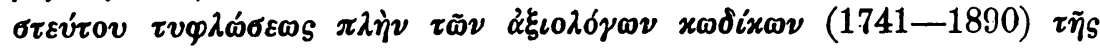

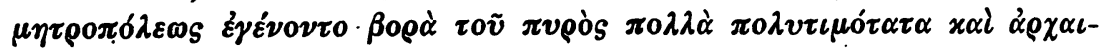

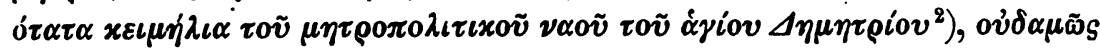

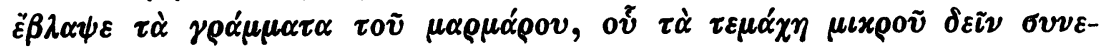

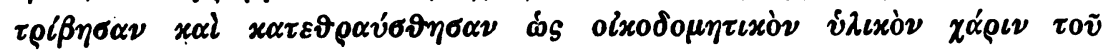

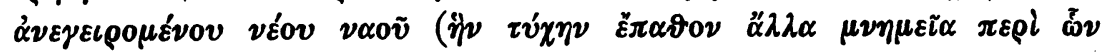

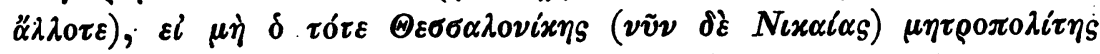

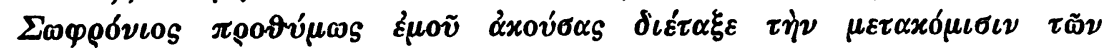

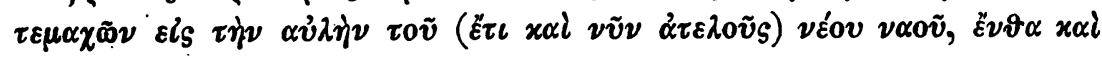

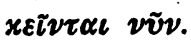

6.

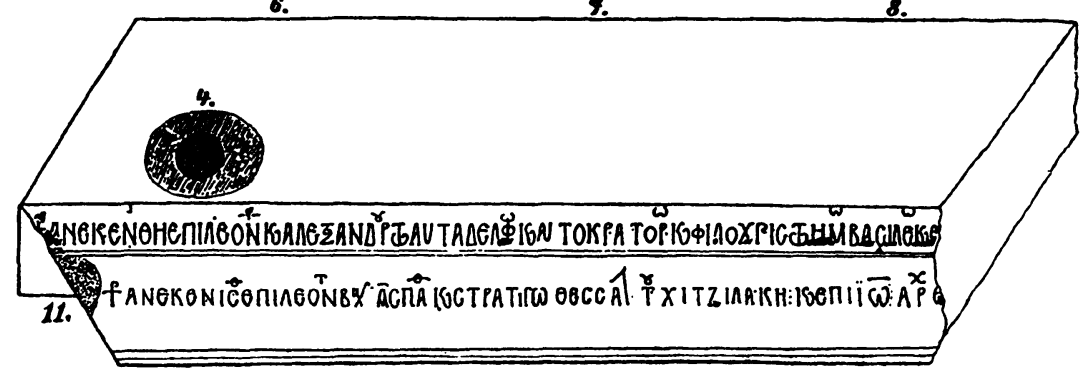

s.

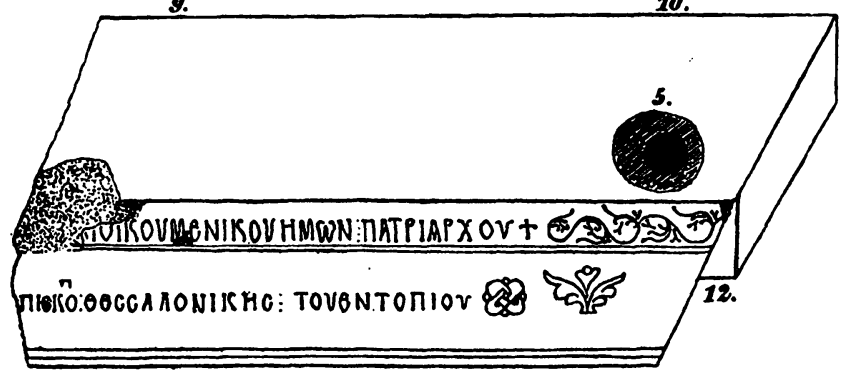

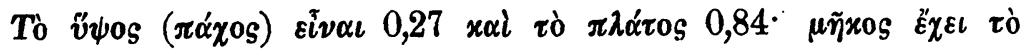

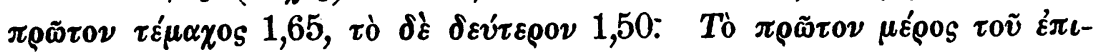

1) Der Brand fing genau in der Nähe der Theodorakirche an.

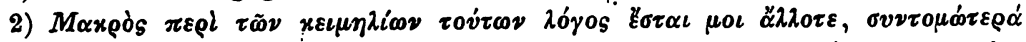

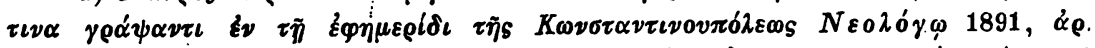

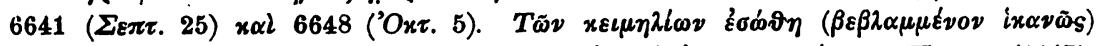

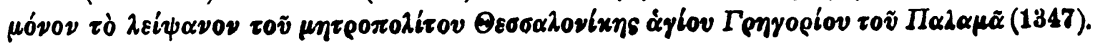




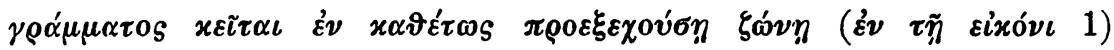

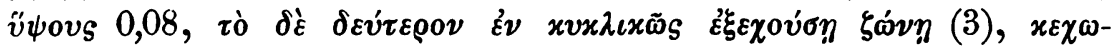

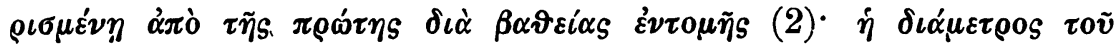

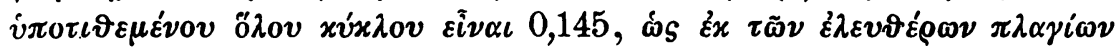

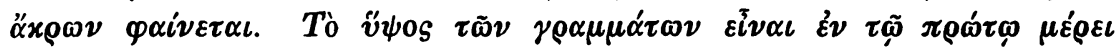

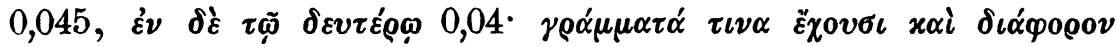

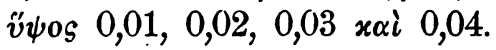

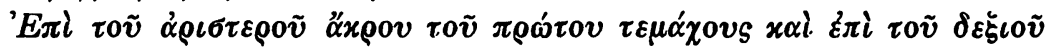

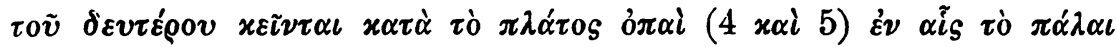

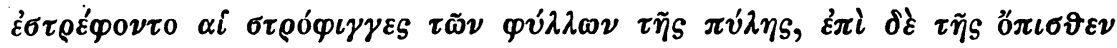

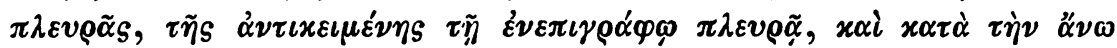

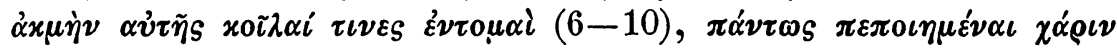

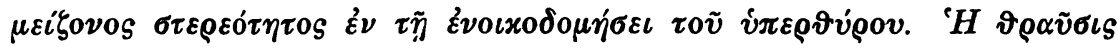

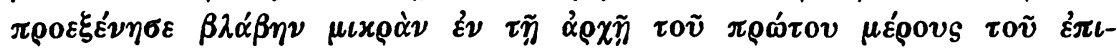

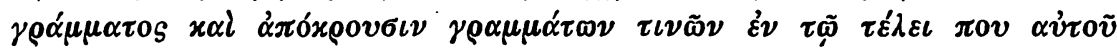

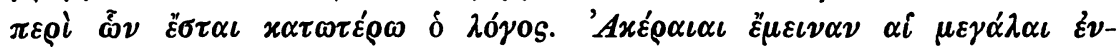

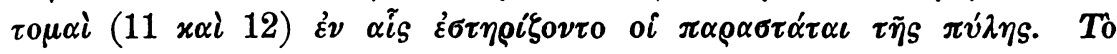

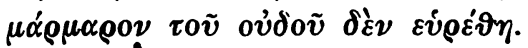

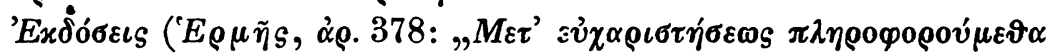

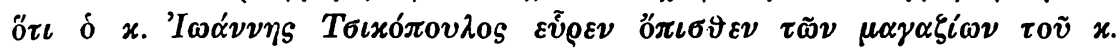

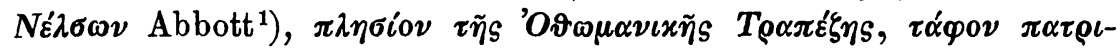

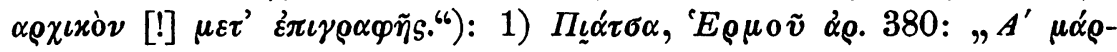

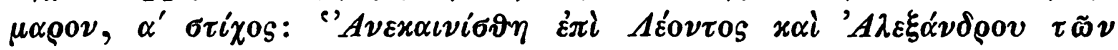

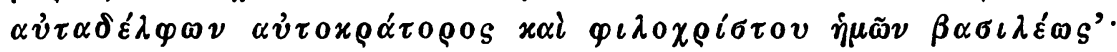

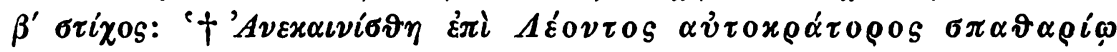

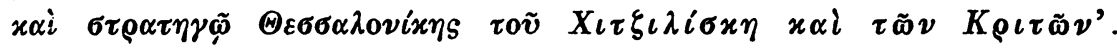

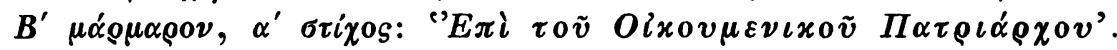

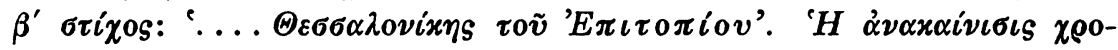

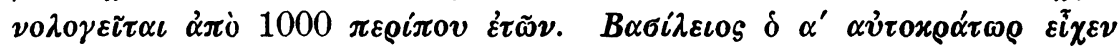

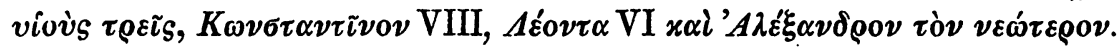

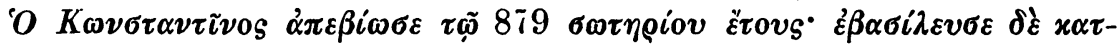

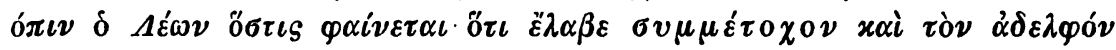

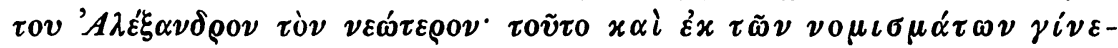

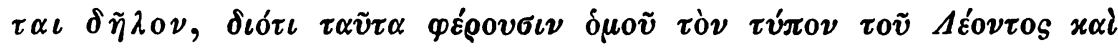

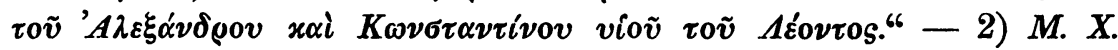

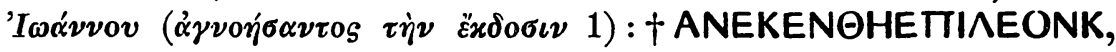

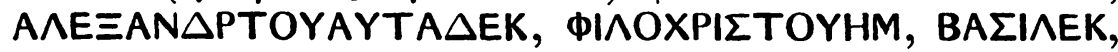
E[TIINIKO $A$ AOYTOY] OIKOYMENIKOYHM $\Omega N \mid T A T P I A P X O Y$

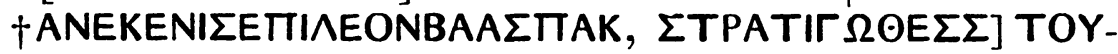

1) Ein anderer als der oben von mir erwähnte Abbott. 


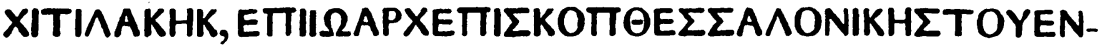

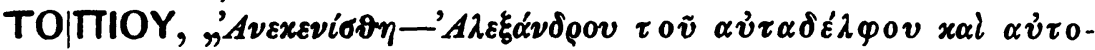

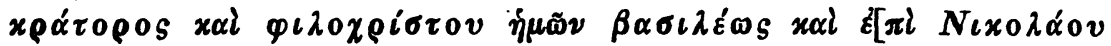

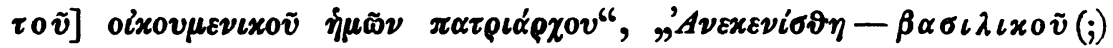

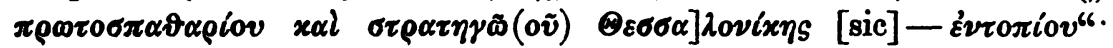

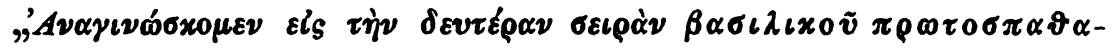

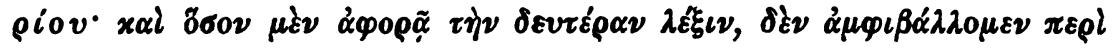

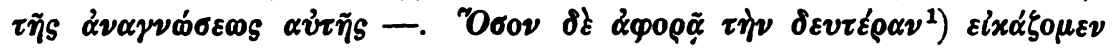

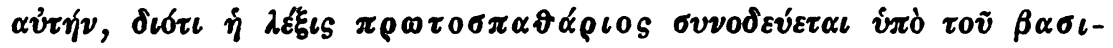
$\lambda \iota x \dot{o} s-. "$

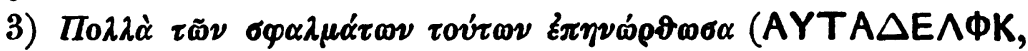

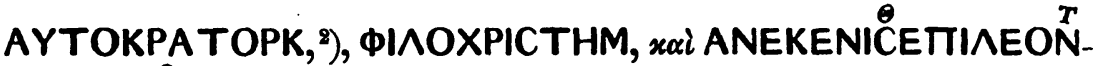

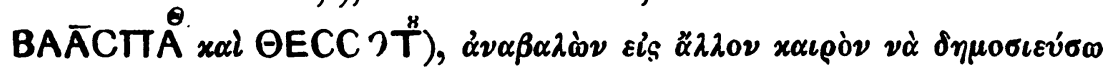

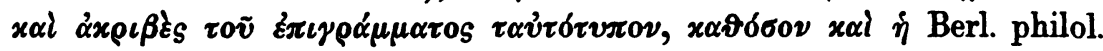

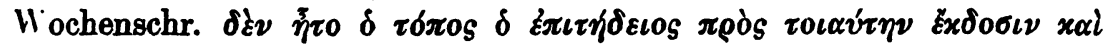


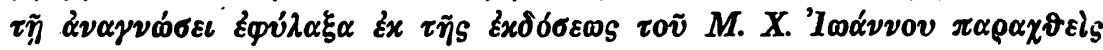

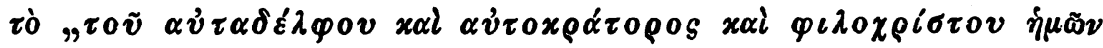

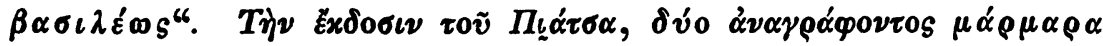

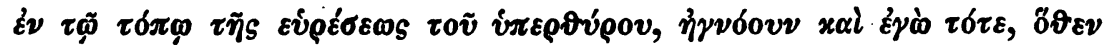

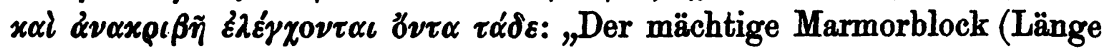

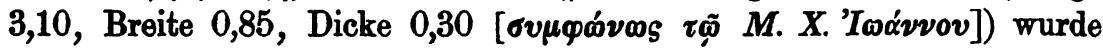
während der Transportierung in die Erzbischofswohnung, wo er sich gegenwärtig befindet, in zwei fast gleich lange Stücke zerbrochen, von denen das erste in BACINEKE und APE endet, das zweite von ........ und TICKO anfängt".

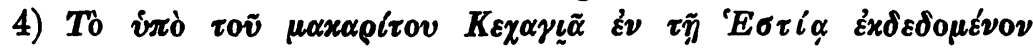

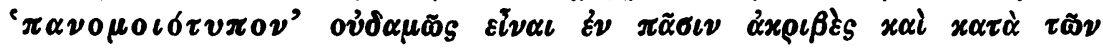

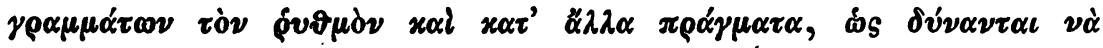

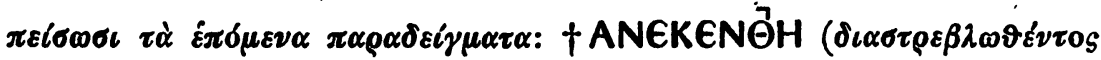

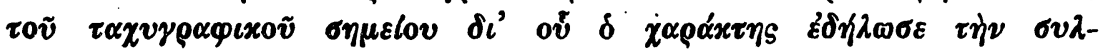

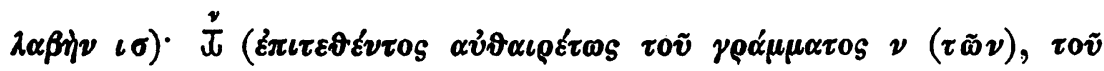

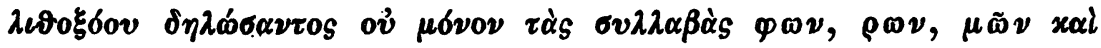

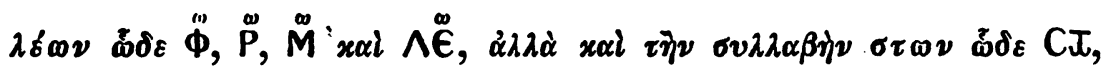

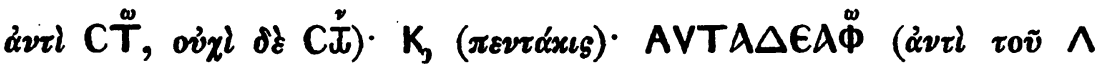

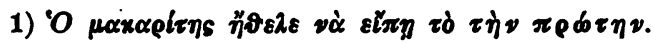

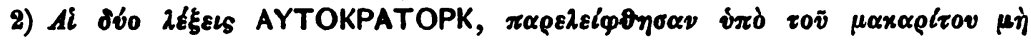

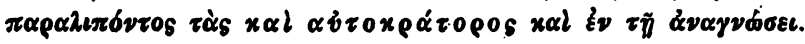




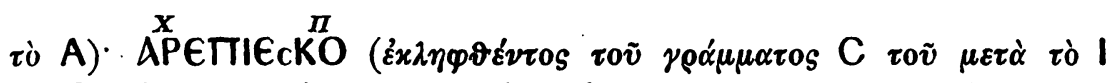

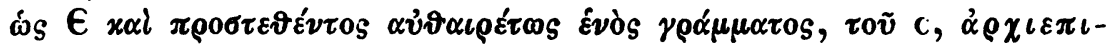

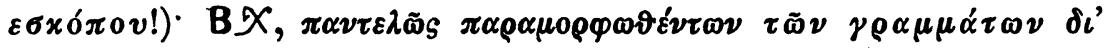

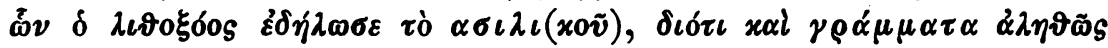

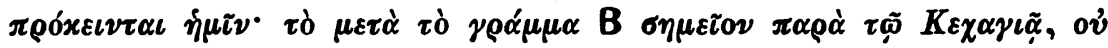

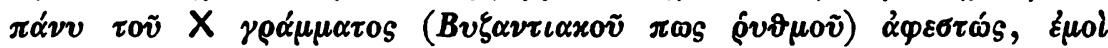

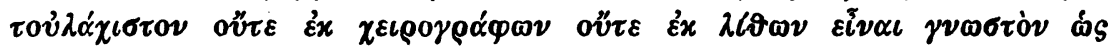

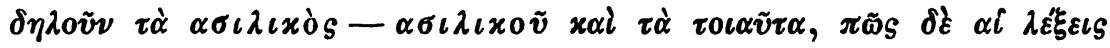

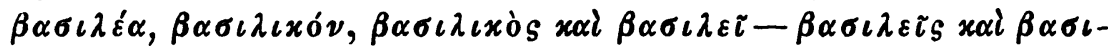

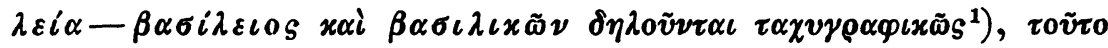
$\delta \iota \delta \alpha ́ \sigma x \varepsilon \iota \dot{\eta} \mu \tilde{\alpha} s \dot{\eta} \tau o \tilde{v}$ Bast Comm. palaeogr., $\sigma \varepsilon \lambda .801$ (Tab. VI 2),

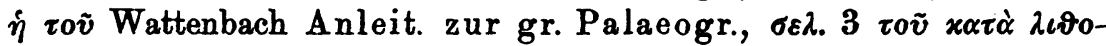

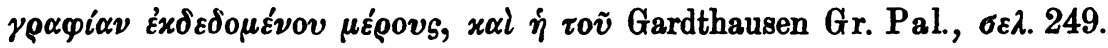

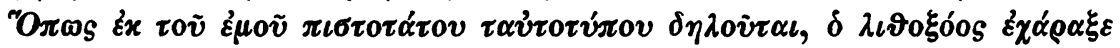

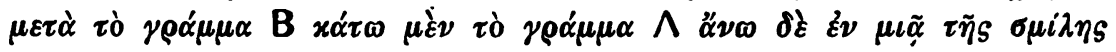

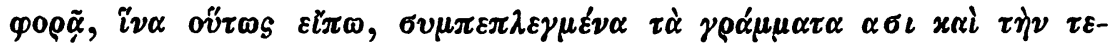

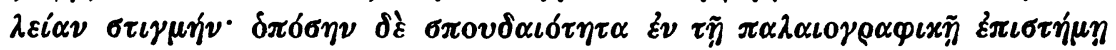

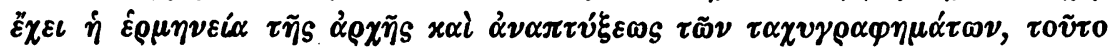

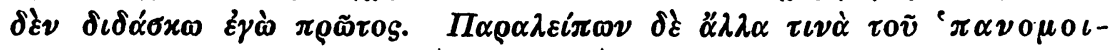

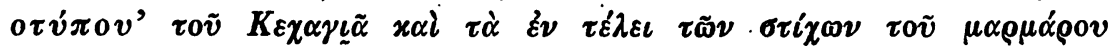

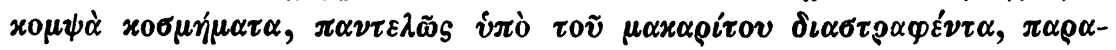

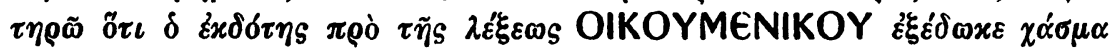

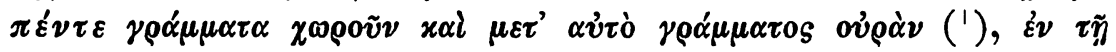

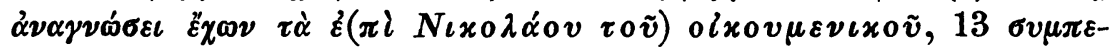

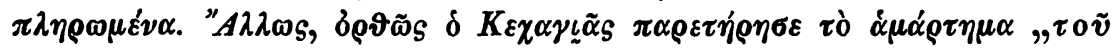

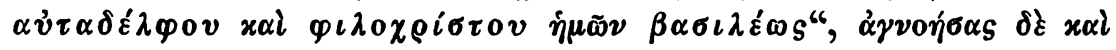

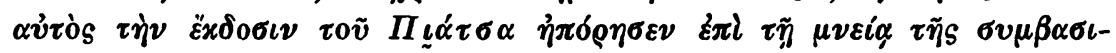

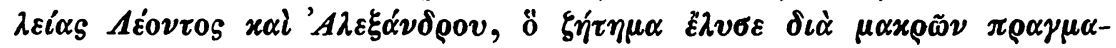

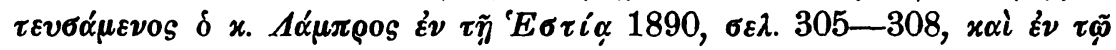

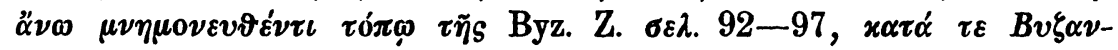

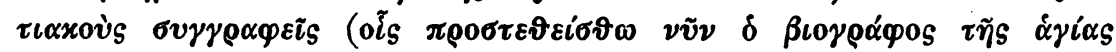

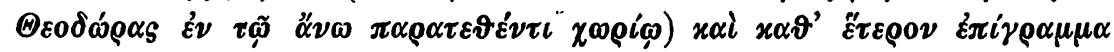

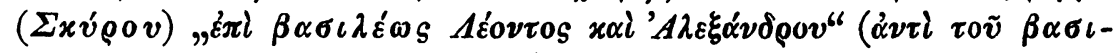

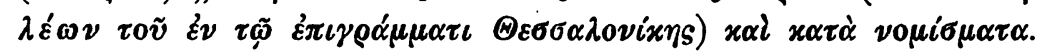

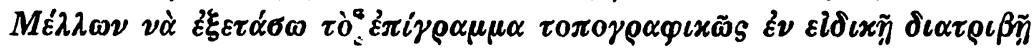

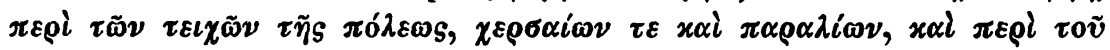

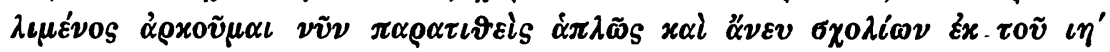

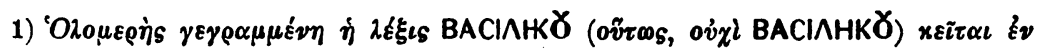

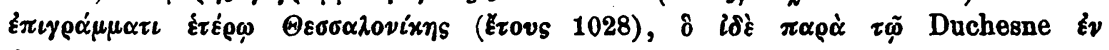

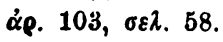




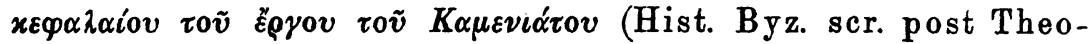

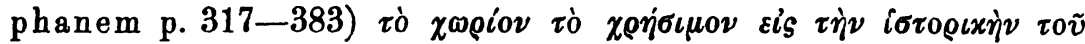

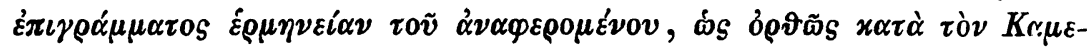

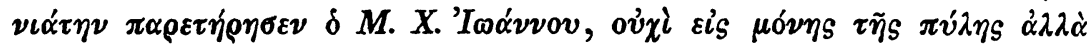

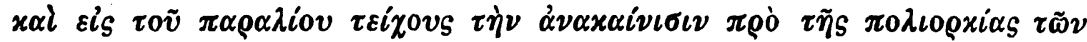

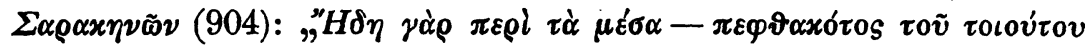

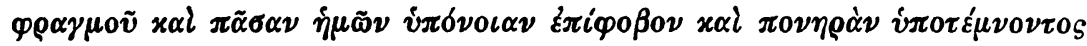

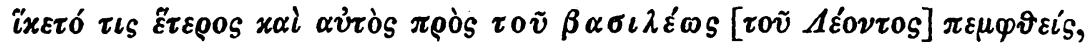

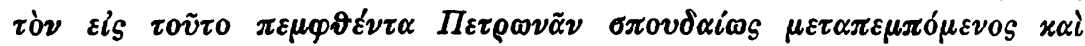

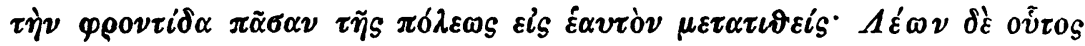

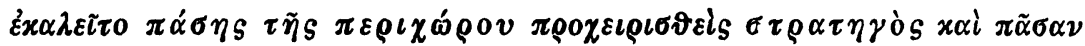

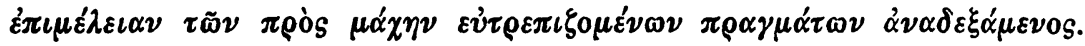

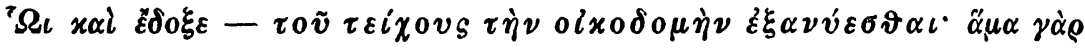

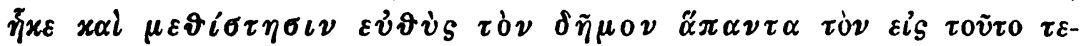

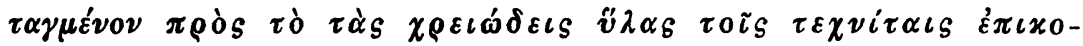

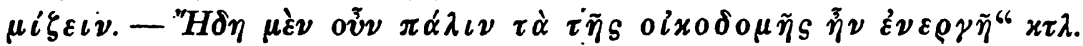

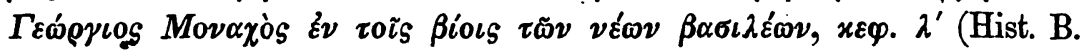

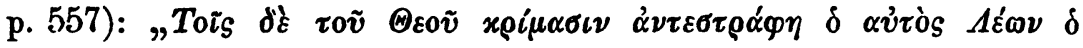

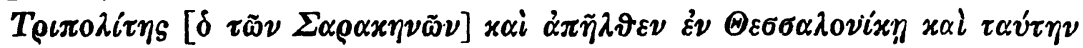

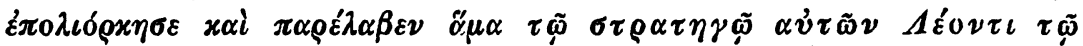

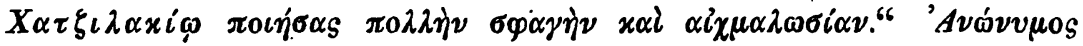

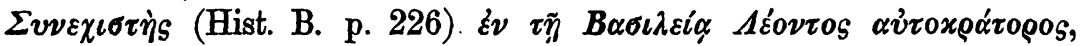

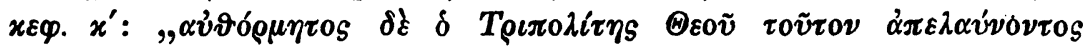

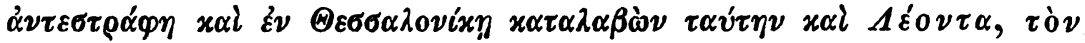

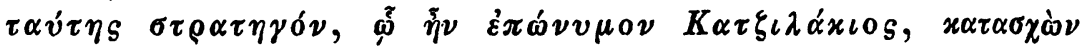

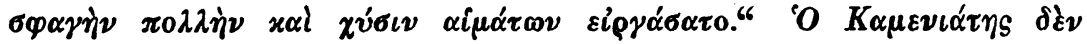

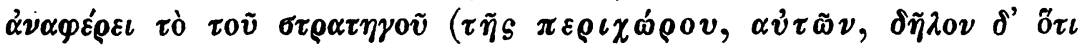

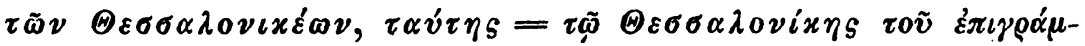

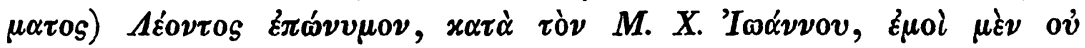

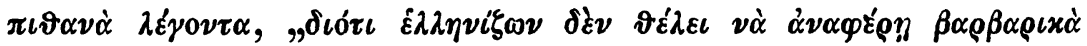

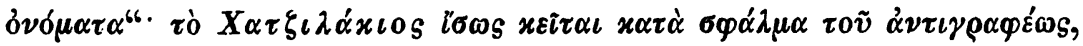

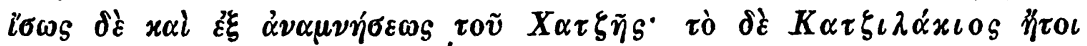

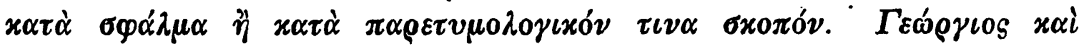

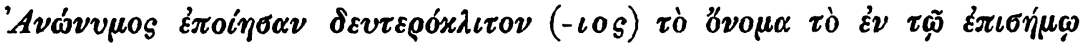

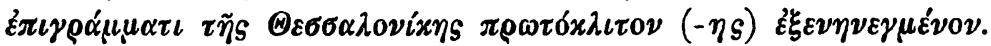

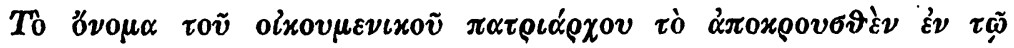

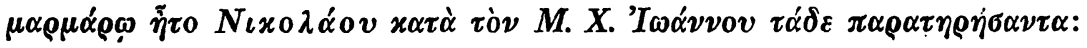

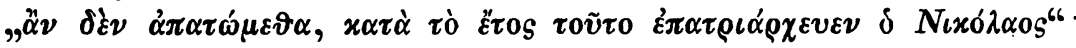

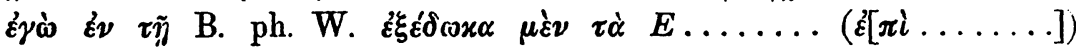
$\pi \alpha \varrho \varepsilon \tau \eta \dot{\rho} \eta \sigma \alpha \delta \delta^{\prime}:$ „Dafs die Ergänzung richtig ist, beweist incert. contin.

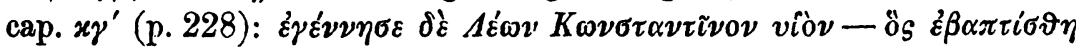




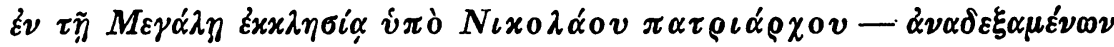

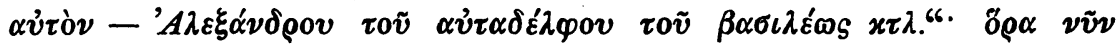

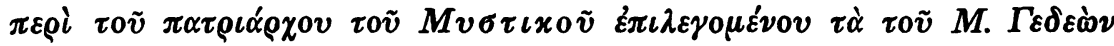

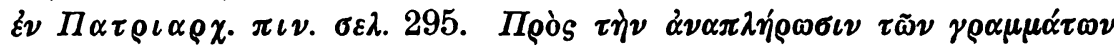

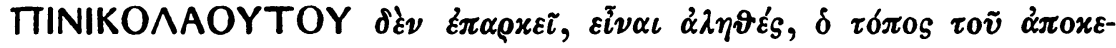

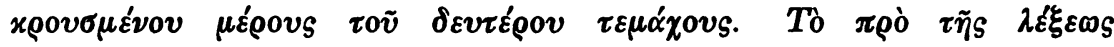

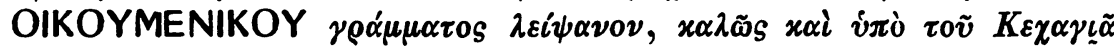

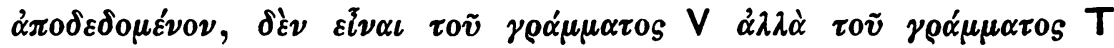

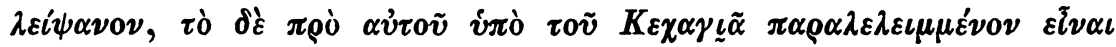

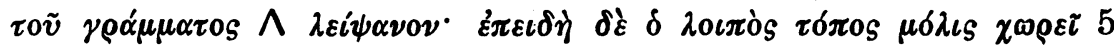

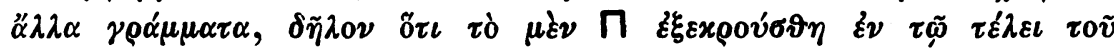

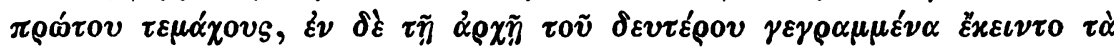
INIKO $\wedge^{8} \mathbf{T}^{8}$.

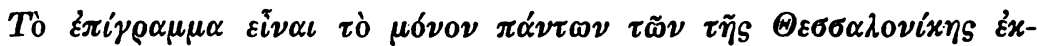

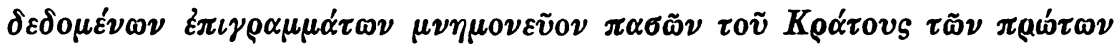

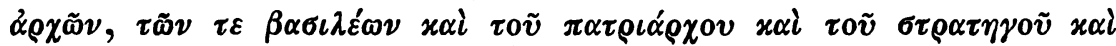

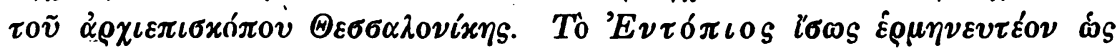

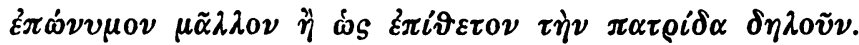

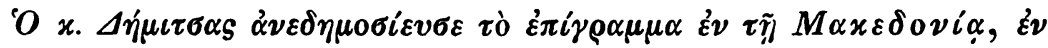

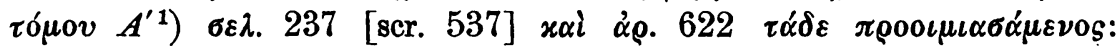

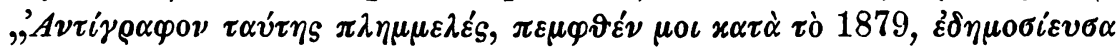

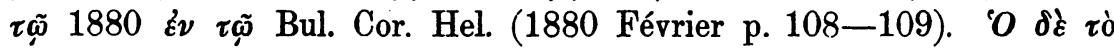

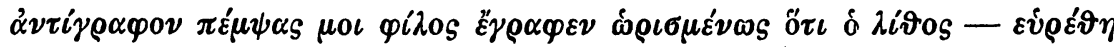

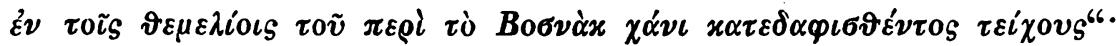

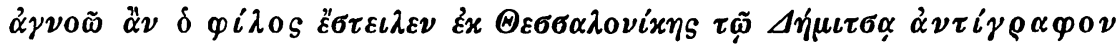

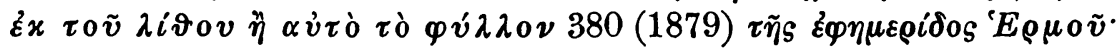

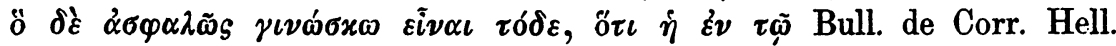

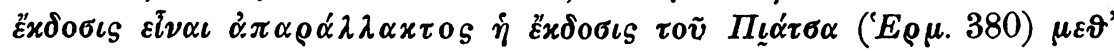

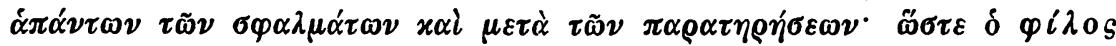

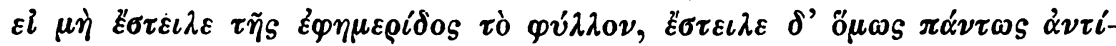


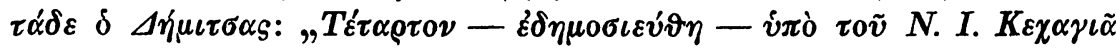

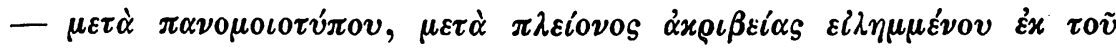

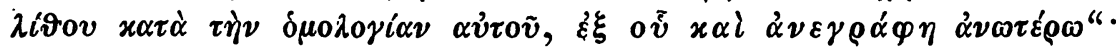

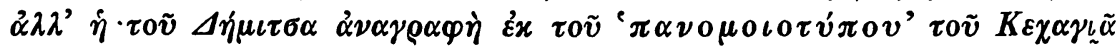

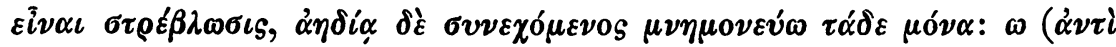

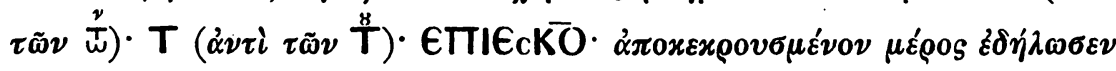

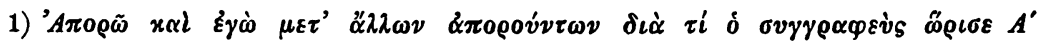

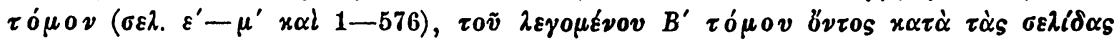

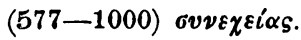


158 I. Abt. P. N. Papageorgin: Zur Vita der hl. Theodora von Thessalonike

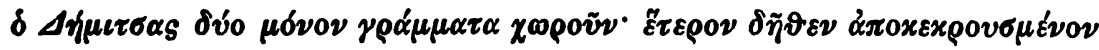

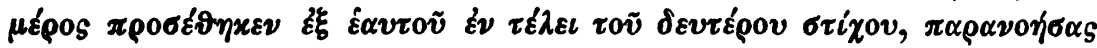

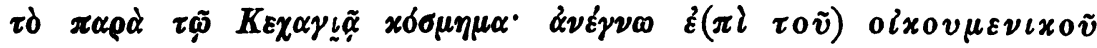

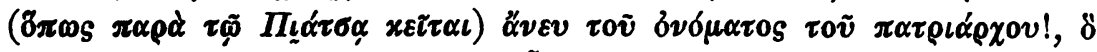

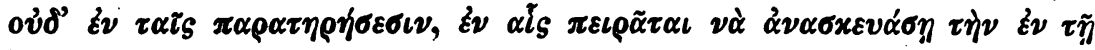

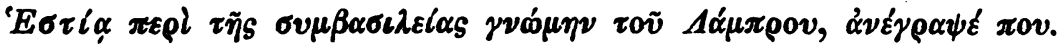

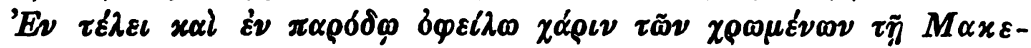

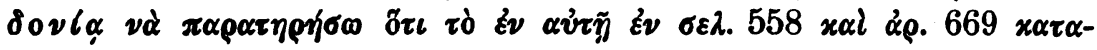

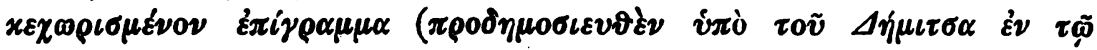

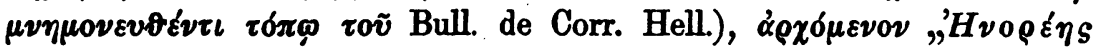

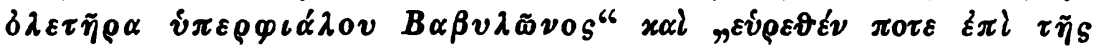

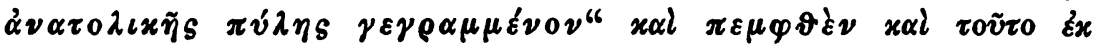

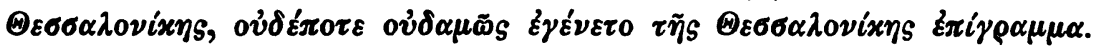

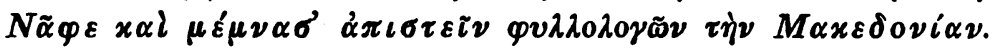

Thessalonike, 25. Nor. 1899.

P. N. Papageorgiu. 